\title{
Classifying superconductivity in an infinite-layer nickelate $\mathrm{Nd}_{0.8} \mathrm{Sr}_{0.2} \mathrm{NiO}_{2}$
}

\author{
E.F. Talantsev ${ }^{\mathrm{a}, \mathrm{b}, *}$ \\ ${ }^{a}$ M.N. Mikheev Institute of Metal Physics, Ural Branch, Russian Academy of Sciences, 18, S. Kovalevskoy St., Ekaterinburg 620108, Russia \\ ${ }^{\mathrm{b}}$ NANOTECH Centre, Ural Federal University, 19 Mira St., Ekaterinburg 620002, Russia
}

\section{A R T I C L E I N F O}

\section{Keywords:}

Superconductivity

Infinite-layer nickelate

Upper critical field

Self-field critical current density

Superconducting energy gap

London penetration depth

\begin{abstract}
A B S T R A C T
Recently Li et al. (2019 Nature 572 624) discovered a new type of oxide superconductor $\mathrm{Nd}_{0.8} \mathrm{Sr}_{0.2} \mathrm{NiO}_{2} \mathrm{with}$ $T_{\mathrm{c}}=14 \mathrm{~K}$. To classify superconductivity in this infinite-layer nickelate experimental upper critical field, $B_{\mathrm{c} 2}(T)$, and the self-field critical current densities, $J_{\mathrm{c}}(\mathrm{sf}, T)$, reported by Li et al. (2019 Nature 572 624), are analysed in assumption of $s$-, $d$-, and $p$-wave pairing symmetries and single- and multiple-band superconductivity. Based on deduced the ground-state superconducting energy gap, $\Delta(0)$, the London penetration depth, $\lambda(0)$, the relative jump in electronic specific heat at $T_{\mathrm{c}}, \Delta C / C$, and the ratio of $2 \Delta(0) / \mathrm{k}_{\mathrm{B}} T_{\mathrm{c}}$, we conclude that $\mathrm{Nd}_{0.8} \mathrm{Sr}_{0.2} \mathrm{NiO}_{2}$ is typeII high-к weak-coupled single-band $s$-wave superconductor.
\end{abstract}

\section{Introduction}

For several decades the term of infinite-layer superconductor was referred to a copper-oxide superconducting compounds, $\mathrm{Sr}_{1-\mathrm{x}} \mathrm{M}_{\mathrm{x}} \mathrm{CuO}_{2}$ ( $\mathrm{M}=\mathrm{La}, \mathrm{Nd}, \mathrm{Ca}, \mathrm{Sr} . .$.$) [1,2], until recently, Li et al. [3] have extended$ this class of unconventional superconductors by the discovery of superconductivity at $T_{\mathrm{c}}=14 \mathrm{~K}$ in $\mathrm{Nd}_{0.8} \mathrm{Sr}_{0.2} \mathrm{NiO}_{2}$ nickelate. Thus, bulk superconducting oxides family, i.e. tungsten bronzes [4], titanates [5], bismuthates [6], cuprates [7], and ruthenates [8] extends by a new nickelate member. Several research groups proposed different models for superconducting state in this compound [9-12], while the exhibiting of the superconducting state in this compound is in a debate [13].

In this paper, to classify superconductivity in this new class of oxide superconductors the temperature-dependent upper critical field, $B_{\mathrm{c} 2}(T)$, and the self-field critical current density, $J_{\mathrm{c}}(\mathrm{sf}, T)$, are analysed within $s$-, $d$-, and p-pairing symmetries. In result, it is shown that infinite-layer $\mathrm{Nd}_{0.8} \mathrm{Sr}_{0.2} \mathrm{NiO}_{2}$ nickelate is weak-coupled single band $s$-wave superconductor.

\section{Models description}

The Ginzburg-Landau theory [14] has two fundamental lengths, one is the coherence length, $\xi(T)$, and the second is London penetration depth, $\lambda(T)$. The ground state coherence length, $\xi(0)$, is given by $[14,15]$ :
$B_{c 2}(0)=\frac{\phi_{0}}{2 \cdot \pi \cdot \xi^{2}(0)}$

where $\phi_{0}=2.068 \cdot 10^{-15} \mathrm{~Wb}$ is magnetic flux quantum, and $B_{\mathrm{c} 2}(0)$ is the ground state upper critical field. For temperature dependent coherence length, $\xi(T)$, several models were proposed [14,15-21]. In this paper, to deduce the ground state coherence length, $\xi(0)$, in infinitelayer $\mathrm{Nd}_{0.8} \mathrm{Sr}_{0.2} \mathrm{NiO}_{2}$ nickelate superconductor, three models are used. The first model was proposed by Gor'kov [16,17] (Gor'kov model):

$B_{c 2}(T)=\frac{\phi_{0}}{2 \cdot \pi \cdot \xi^{2}(0)} \cdot\left(\frac{1.77-0.43 \cdot\left(\frac{T}{T_{c}}\right)^{2}+0.07 \cdot\left(\frac{T}{T_{c}}\right)^{4}}{1.77}\right) \cdot\left[1-\left(\frac{T}{T_{c}}\right)^{2}\right]$

The second model was proposed by Baumgartner et al. [20] (BWHH):

$B_{c 2}(T)=\frac{\phi_{0}}{2 \cdot \pi \cdot \xi^{2}(0)} \cdot\left(\frac{\left(1-\frac{T}{T_{c}}\right)-0.153 \cdot\left(1-\frac{T}{T_{c}}\right)^{2}-0.152 \cdot\left(1-\frac{T}{T_{c}}\right)^{4}}{0.693}\right)$

And the third model was proposed recently in our recent report [21]:

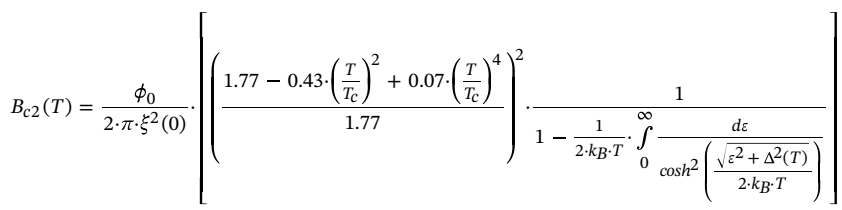

\footnotetext{
* Address: M.N. Mikheev Institute of Metal Physics, Ural Branch, Russian Academy of Sciences, 18, S. Kovalevskoy St., Ekaterinburg 620108, Russia.

E-mail address: evgeny.talantsev@imp.uran.ru.
} 
where $k_{\mathrm{B}}$ is Boltzmann constant, and $\Delta(T)$ is the temperature-dependent superconducting gap, for which analytical expression was given by Gross et al. [22]:

$\Delta(T)=\Delta(0) \cdot \tanh \left[\frac{\pi \cdot k_{B} \cdot T_{c}}{\Delta(0)} \cdot \sqrt{\eta \cdot \frac{\Delta C}{C} \cdot\left(\frac{T_{c}}{T}-1\right)}\right]$

where $\Delta(0)$ is the ground state energy gap amplitude, $\Delta C / C$ is the relative jump in electronic specific heat at $T_{\mathrm{c}}, \eta=2 / 3$ for $s$-wave superconductors [22].

Thus, $\xi(0)$ and $T_{\mathrm{c}}$ can be obtained by fitting experimental $B_{\mathrm{c} 2}(T)$ data to Eqs. (2)-(4). In addition, $\Delta C / C, \Delta(0)$ and, thus, the ratio of $\frac{2 \Delta(0)}{k_{D} T_{T}}$, can be deduced as free-fitting parameters by fitting experimental $B_{\mathrm{c} 2}(T)$ data to Eq. (4). More details about the procedures can be found elsewhere [23].

There is an alternative way to deduce $\Delta(0), \Delta C / C, T_{\mathrm{c}}$ and $\frac{2 \Delta(0)}{k_{B} T_{\mathrm{c}}}$ by the fit of experimental self-field critical current density, $J_{\mathrm{c}}(\mathrm{sf}, T)$, to universal equation, which is for thin-film superconductors reduced to simple form $[23,24]$ :

$J_{c}($ sf, $T)=\frac{\phi_{0}}{4 \pi \mu_{0}} \cdot \frac{\ln \left(1+\sqrt{2} \cdot \frac{\lambda(0)}{\xi(0)}\right)}{\lambda^{3}(T)}$

where $\mu_{0}=4 \pi \times 10^{-7} \mathrm{H} / \mathrm{m}$ is the magnetic permeability of free space, and the London penetration depth, $\lambda(T)$, is given by:

$\lambda(T)=\frac{\lambda(0)}{\sqrt{1-\frac{1}{2 \cdot k_{B} \cdot T} \cdot \int_{0}^{\infty} \frac{d \varepsilon}{\cosh ^{2}\left(\frac{\sqrt{\varepsilon^{2}+\Delta^{2}(T)}}{2 \cdot k_{B} \cdot T}\right)}}}$

for $s$-wave superconductors, where $\Delta(T)$ is given by Eq. (5) $[22,25]$.

$\lambda(T)=\frac{\lambda(0)}{\sqrt{1-\frac{1}{2 \cdot k_{B} \cdot T} \cdot \int_{0}^{2 \pi} \cos ^{2}(\theta) \cdot\left(\int_{0}^{\infty} \frac{d \varepsilon}{\cosh ^{2}\left(\frac{\sqrt{\varepsilon^{2}+\Delta^{2}(T, \theta)}}{2 \cdot k_{B} \cdot T}\right)}\right) \cdot d \theta}}$

for $d$-wave superconductors, where the superconducting energy gap, $\Delta(T, \theta)$, is given by $[22,25]$ :

$\Delta(T, \theta)=\Delta_{m}(T) \cdot \cos (2 \theta)$

where $\Delta_{\mathrm{m}}(T)$ is the maximum amplitude of the $k$-dependent $d$-wave gap given by Eq. (5), $\theta$ is the angle around the Fermi surface subtended at $(\pi, \pi)$ in the Brillouin zone (details can be found elsewhere $[22,25,26]$ ). In Eq. (9) the value of $\eta=7 / 5[22,25,26]$.

1. And $p$-wave symmetry $[22,25]$, which only recently was tested to fit critical current densities in superconductors $[22,25]$ :

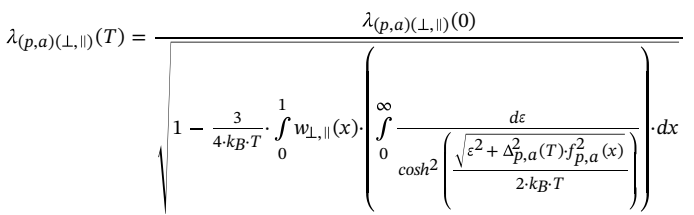

where subscripts $p, a, \perp$, and $\|$ designate polar, axial, perpendicular and parallel cases respectively. For this symmetry, the gap function is given by $[22,25]$ :

$\Delta(\hat{\boldsymbol{k}}, T)=\Delta(T) \cdot f(\hat{\boldsymbol{k}}, \hat{\boldsymbol{l}})$

where, $\Delta(T)$ is the superconducting gap amplitude, $\boldsymbol{k}$ is the wave vector, and $l$ is the gap axis. Thus, temperature dependence of $\lambda(T)$ is determined by mutual orientation of the vector potential, $\boldsymbol{A}$, and the gap axis, $\boldsymbol{l}$, which is for transport current experiment just the orientation of the crystallographic axes of the film compared with the direction of the electric current. There are two distinctive orientations, $A \perp l$ (when $\boldsymbol{A}$ is perpendicular to $\boldsymbol{l}$ ) and polar $\mathbf{A} \| \boldsymbol{l}$ (when $\boldsymbol{A}$ is parallel to $\boldsymbol{l}$ ) [22,25]. More details can be found elsewhere $[22,25,26])$. The function of $w_{\perp, \|}(x)$ in Eq. (10) is:

$w_{\perp}(x)=\left(1-x^{2}\right) / 2$

and

$w_{\|}(x)=x^{2}$

and the gap amplitude in Eq. (11) is just Eq. (5), but $\eta$ is given by [25]:

$\eta_{p, a}=\frac{2}{3} \cdot \frac{1}{\int_{0}^{1} f_{p, a}^{2}(x) \cdot d x}$

where

$f_{p}(x)=x ;$ polar configuration

$f_{a}(x)=\sqrt{1-x^{2}} ;$ axial configuration

More details about the $J_{\mathrm{c}}(\mathrm{sf}, T)$ analysis for $p$-wave symmetry can be found elsewhere $[26,27]$.

By substituting Eqs. (5), (7)-(13) in Eq. (6), one can fit experimental $J_{\mathrm{c}}(\mathrm{sf}, T)$ data to $s$-, $d$-, $p$-wave gap symmetries to deduce $\lambda(0), \Delta(0), \Delta C /$ $C, T_{\mathrm{c}}$ and $\frac{2 \Delta(0)}{k_{B} T_{\mathrm{c}}}$ as free-fitting parameters. This approach is recently applied for wide range of thin film unconventional superconductors [23,24,26-32].

\section{$B_{\mathrm{c} 2}(T)$ analysis}

There are several criteria to define $B_{\mathrm{c} 2}(T)$ from experimental $R(T)$ curves. In this paper to define $B_{\mathrm{c} 2}(T)$ we use the criterion of $3 \%$ of normal state resistance, $R_{\text {norm }}(T)$, for $R(T)$ curves of $\mathrm{Nd}_{0.8} \mathrm{Sr}_{0.2} \mathrm{NiO}_{2}$ presented in Fig. 4 (a) by Li et al. [3]. The fits of $B_{\mathrm{c} 2}(T)$ data to three models are shown in Fig. 1. It can be seen that $\xi(0)$ values deduced by three models are close to each other and following analysis of $J_{\mathrm{c}}(\mathrm{sf}, T)$ will be utilized an average value of:

$\xi(0)=5.7 \pm 0.3 \mathrm{~nm}$

This deduced value for $\xi(0)$ is in reasonable agreement with $\xi(0)=4.5 \mathrm{~nm}$ reported by Jovanović et al. [33] for copper-oxide-based infinite layer counterpart of $\mathrm{La}_{1-\mathrm{x}} \mathrm{Sr}_{\mathrm{x}} \mathrm{CuO}_{2}$.

Deduced values by the fit to Eq. (4):

$\frac{2 \Delta(0)}{k_{B} T_{c}}=3.5 \pm 0.3$

$\frac{\Delta \mathrm{C}}{C}=1.5 \pm 0.2$

are, within uncertainties, equal to BCS [34] weak-coupling limits of 3.53 and 1.43 respectively, and the former deduced value is equal to recently deduced value of:

$\frac{2 \Delta(0)}{k_{B} T_{c}}=3.51 \pm 0.05$

for $s$-wave oxide superconductor of $\mathrm{Ba}_{0.51} \mathrm{~K}_{0.49} \mathrm{BiO}_{3}$ [35].

It should be noted that there is no sign in experimental $B_{\mathrm{c} 2}(T)$ data that $\mathrm{Nd}_{0.8} \mathrm{Sr}_{0.2} \mathrm{NiO}_{2}$ exhibits two superconducting band state, which can be seen as sharp enhancement in amplitude of $B_{\mathrm{c} 2}(T)$ at critical temperature of the second superconducting band opening (see for details Ref. 36).

\section{$J_{c}(\mathrm{sf}, T)$ analysis}

The critical current density, $J_{\mathrm{c}}$, is defined as the lowest, detectable in experiment, value of electric power dissipation in a superconductor on electric current flow. For available $E(I)$ curves presented by Li et al. [3] in their Fig. 3(f), the critical current density at self-field condition 


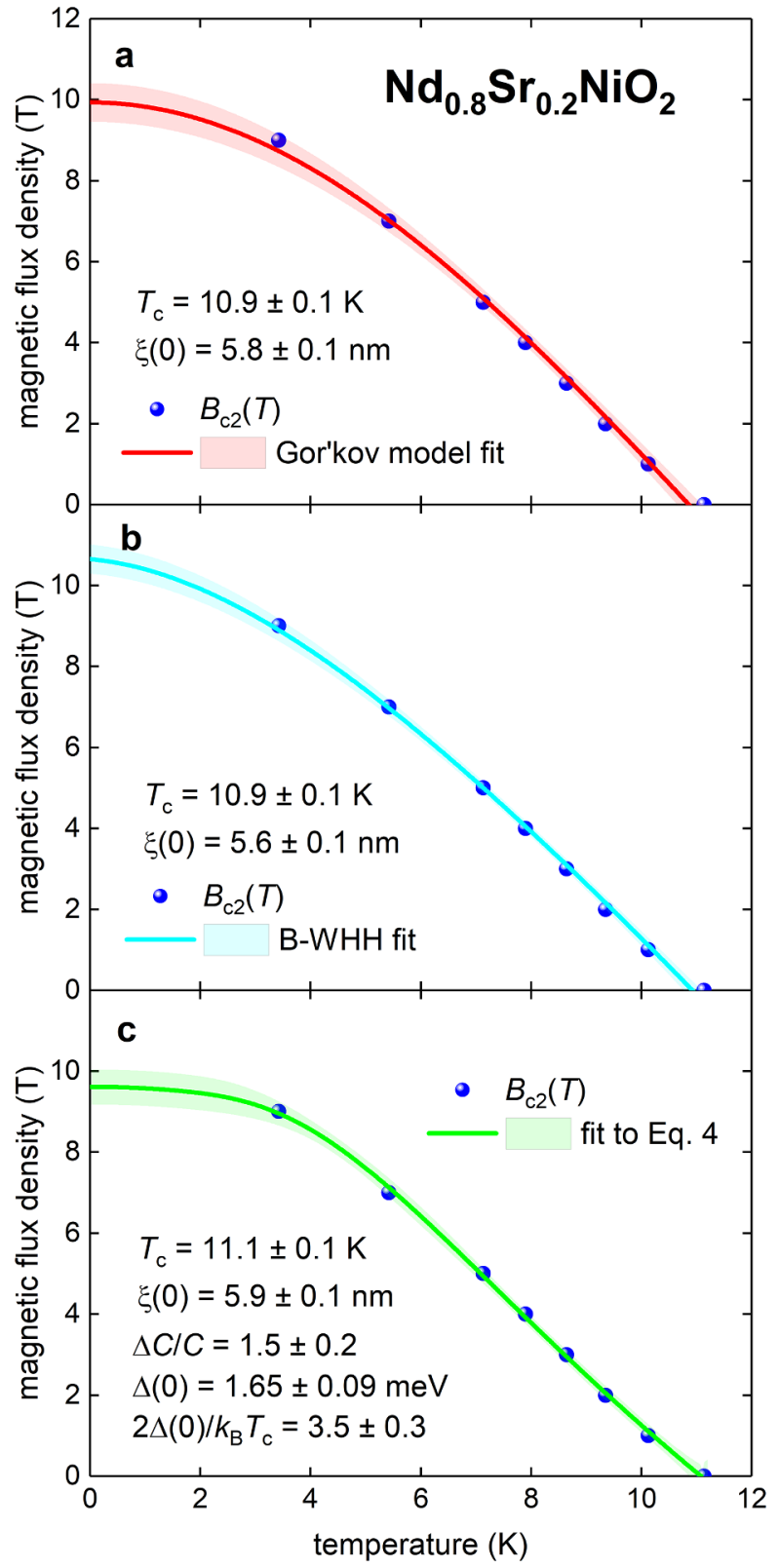

Fig. 1. The upper critical field, $B_{\mathrm{c} 2}(T)$, of $\mathrm{Nd}_{0.8} \mathrm{Sr}_{0.2} \mathrm{NiO}_{2}$ (reported by $\mathrm{Li}$ et al. [3]) and data fits to three models (Eqs. (2)-(4)). (a) Fit to Gor'kov model, the fit quality is $R=0.995$. (b) Fit to B-WHH, $R=0.998$. (c) Fit to Eq. (4), $R=0.9993 .95 \%$ confidence bars are shown.

(when no external magnetic field is applied), $J_{\mathrm{c}}(\mathrm{sf}, T)$, can be defined at the lowest value of electric field of $E_{\mathrm{c}}=3 \mathrm{~V} / \mathrm{cm}$. Experimental $J_{\mathrm{c}}(\mathrm{sf}, T)$ deduced by this $E_{\mathrm{c}}$ criterion and the fit to single band $s$-wave model (i.e., Eqs. (6) and (7) for which $\xi(0)=5.7 \mathrm{~nm}$ was fixed) are shown in Fig. 2(a). It can be seen that the fit is excellent, and deduced superconducting parameters (Fig. 2(a) and Table 1) are within BCS weakcoupling limits.

Deduced $\lambda(0)=740 \pm 3 \mathrm{~nm}$ is similar to $\lambda(0)=690-850 \mathrm{~nm}$ measured for samples possessing maximal $T_{\mathrm{c}}$ values for cuprate counterpart $\mathrm{La}_{1-\mathrm{x}} \mathrm{Sr}_{\mathrm{x}} \mathrm{CuO}_{2}$ [37].

By utilizing deduced $\lambda(0)$ value the Ginzburg-Landau parameter $\kappa=\frac{\lambda(0)}{\xi(0)}=130$ which is similar to $\mathrm{La}_{1-\mathrm{x}} \mathrm{Sr}_{\mathrm{x}} \mathrm{CuO}_{2}[33,37]$ and this value is at the upper-level range for other cuprates and unconventional superconductors [15,23,24,26,38-43].

Alternatively, $d$ - and $p$-wave superconducting gap symmetries can be considered. The fits to $d$-wave symmetry, as well as to polar $\mathbf{A} \perp l$ and

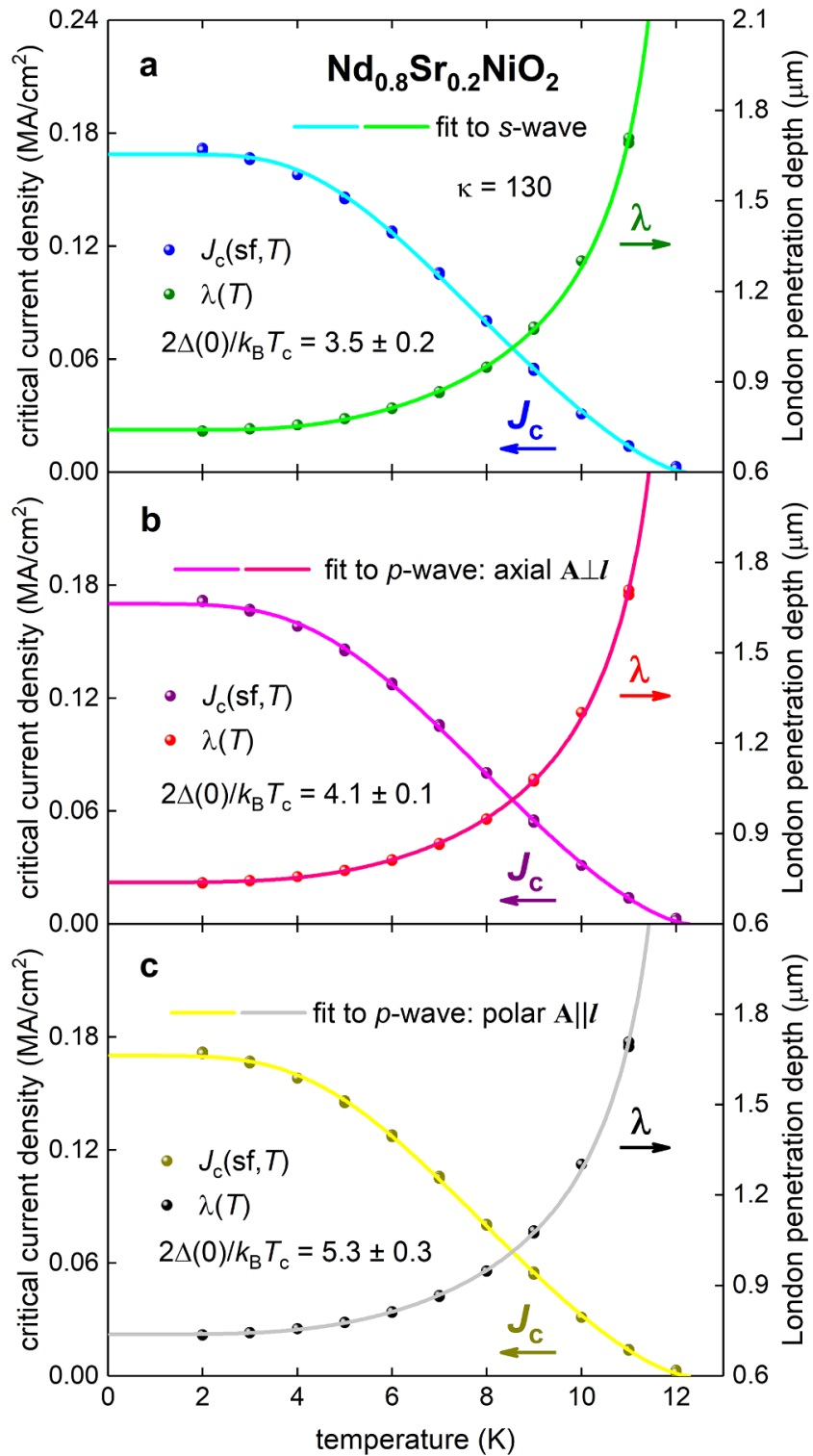

Fig. 2. The self-field critical current density, $J_{\mathrm{c}}(\mathrm{sf}, T)$, for $\mathrm{Nd}_{0.8} \mathrm{Sr}_{0.2} \mathrm{NiO}_{2}$ thin film with raw data processed from the work of Li et al. [3] and data fits to three single-band models for which deduced parameters are close to weak-coupling BCS limits. For all models $\xi(0)=5.7 \mathrm{~nm}$ was used. (a) $s$-wave fit, $\lambda(0)=740 \pm 3 \mathrm{~nm}, T_{\mathrm{c}}=12.2 \pm 0.1 \mathrm{~K}$, the goodness of fit $R=0.995$; (b) $p$ wave axial $\mathrm{A} \perp \boldsymbol{l}$ fit, $\lambda(0)=738 \pm 2 \mathrm{~nm}, T_{\mathrm{c}}=12.3 \pm 0.1 \mathrm{~K}, R=0.997$; (c) $p$ wave polar A $\| \boldsymbol{l}$ fit, $\lambda(0)=735 \pm 2 \mathrm{~nm}, T_{\mathrm{c}}=12.4 \pm 0.1 \mathrm{~K}, R=0.9990$. Other deduced parameters are listed in Table 1.

axial $\mathbf{A} \| \boldsymbol{l}$ of $p$-wave are shown in Fig. 3. However, fits to these symmetries reveal very large $\frac{2 \Delta(0)}{k_{B} T_{c}}$ values (Table 1 and Fig. 3). And based on this, these three symmetries can be excluded from further consideration.

The cases of polar $\mathbf{A} \| \boldsymbol{l}$ and axial $\mathbf{A} \perp \boldsymbol{l}$ gap symmetries are still hypothetically possible (Table 1 ), and $J_{\mathrm{c}}(\mathrm{sf}, T)$ fits to these models are shown in Fig. 2(b, c) respectively, however, for given experimental conditions (i.e. epitaxial $c$-axis oriented thin film) expected geometry is polar $\mathbf{A} \perp \boldsymbol{l}[21]$, but for this geometry deduced $\frac{2 \Delta(0)}{k_{B} T_{c}} \cong 1950$ (Fig. 3(c)) which does not have physical meaningful value.

It should be stressed that there is no sign for two-band superconductivity in $\mathrm{Nd}_{0.8} \mathrm{Sr}_{0.2} \mathrm{NiO}_{2}$ which usually can be detected by a sharp enhancement in $B_{\mathrm{c} 2}(T)$ and $J_{\mathrm{c}}(\mathrm{sf}, T)$ at critical temperature of the second band opening $[24,36]$.

It is important to note that there are strong experimental evidences [44] that cuprate infinite layer superconductor, $\mathrm{Sr}_{0.90} \mathrm{La}_{0.10} \mathrm{CuO}_{2}$, which 


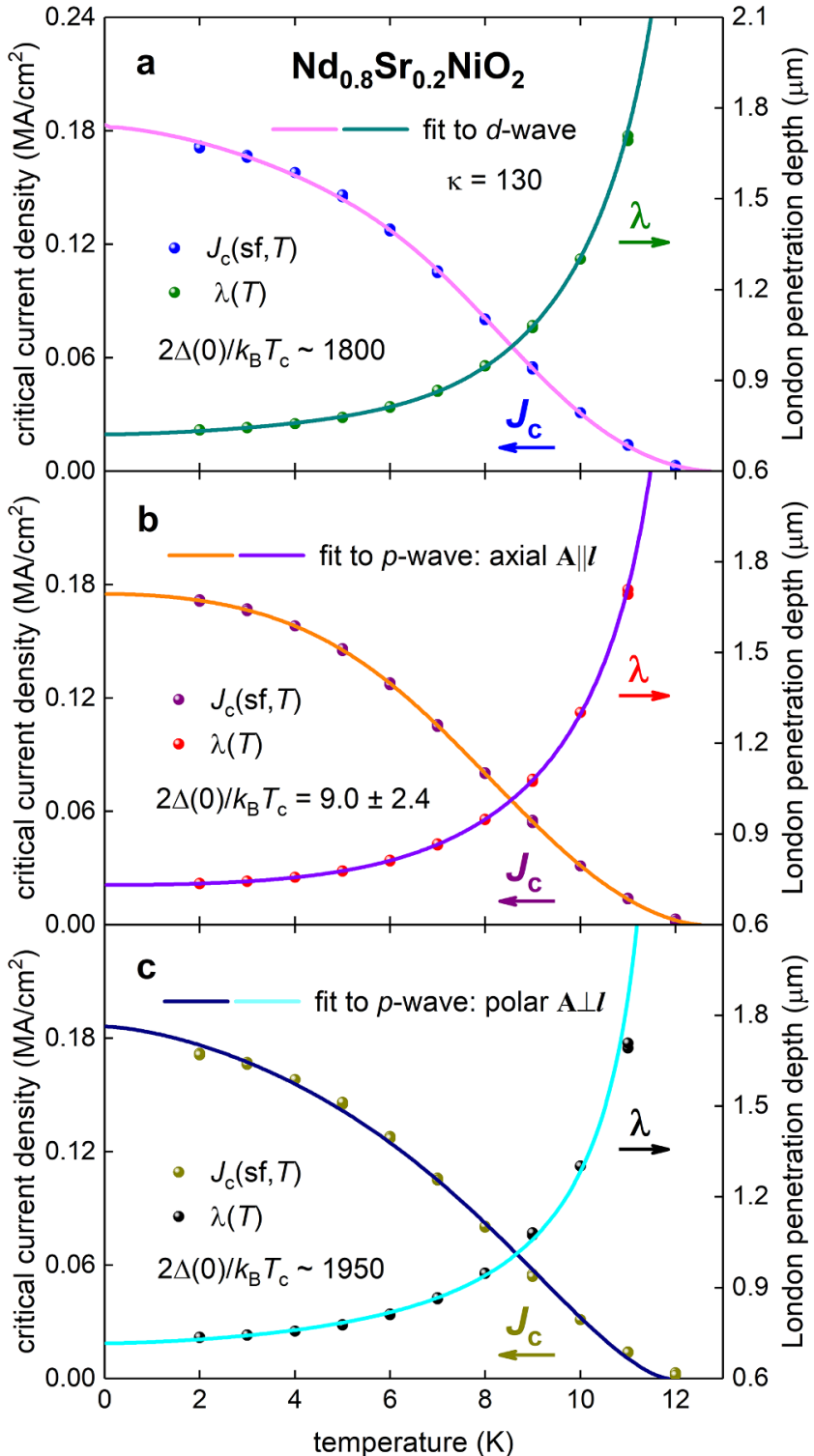

Fig. 3. The self-field critical current density, $J_{\mathrm{c}}(\mathrm{sf}, T)$, for $\mathrm{Nd}_{0.8} \mathrm{Sr}_{0.2} \mathrm{NiO}_{2}$ thin film with raw data processed from the work of Li et al. [3] and data fit to three single-band models for which deduced parameters are very different from weak-coupling BCS limits. Ground state superconducting coherence length of $\xi(0)=5.7 \mathrm{~nm}$ was used. (a) $d$-wave fit, $\lambda(0)=721 \pm 4 \mathrm{~nm}$, $T_{\mathrm{c}}=12.7 \pm 0.2 \mathrm{~K}$, the goodness of fit $R=0.997$; (b) $p$-wave axial $\mathbf{A} \| \boldsymbol{l}$ fit, $\lambda(0)=738 \pm 2 \mathrm{~nm}, T_{\mathrm{c}}=12.53 \pm 0.05 \mathrm{~K}, R=0.9996$; (c) $p$-wave polar $\mathrm{A} \perp l$ fit, $\lambda(0)=716 \pm 12 \mathrm{~nm}, T_{\mathrm{c}}=11.9 \pm 0.2 \mathrm{~K}, R=0.986$. Other deduced parameters are listed in Table 1.

is perhaps the closest structural counterpart of $\mathrm{Nd}_{0.8} \mathrm{Sr}_{0.2} \mathrm{NiO}_{2}$ in the cuprate superconductors family $[1,2,7,45,46]$ is $s$-wave superconductor [44]. In this regard, our finding that $\mathrm{Nd}_{0.8} \mathrm{Sr}_{0.2} \mathrm{NiO}_{2}$ is $s$-wave superconductor is rather convincing, because it shows a similarity with cuprate superconductors, rather than to be a contradiction with cuprate superconductors, where $d$-wave superconductivity exhibits in materials with charge reservoir atomic layers.

To further demonstrate that available experimental data for $\mathrm{Nd}_{0.8} \mathrm{Sr}_{0.2} \mathrm{NiO}_{2}$ superconductor is inconsistent with $d$-wave gap symmetry in Fig. 4 we show the fit of $J_{\mathrm{c}}(\mathrm{sf}, T)$ to $d$-wave model for which the ratio of $\frac{2 \Delta(0)}{k_{B} T_{c}}$ was fixed to three different values: (a) to the weak-coupling limit, $\frac{2 \Delta(0)}{k_{B} T_{C}}=4.28$ [25,47], of $d$-wave superconductors (Fig. 4a); (b) to $\frac{2 \Delta(0)}{k_{B} T_{c}}=6.0$ which can be considered as very strong-coupling case

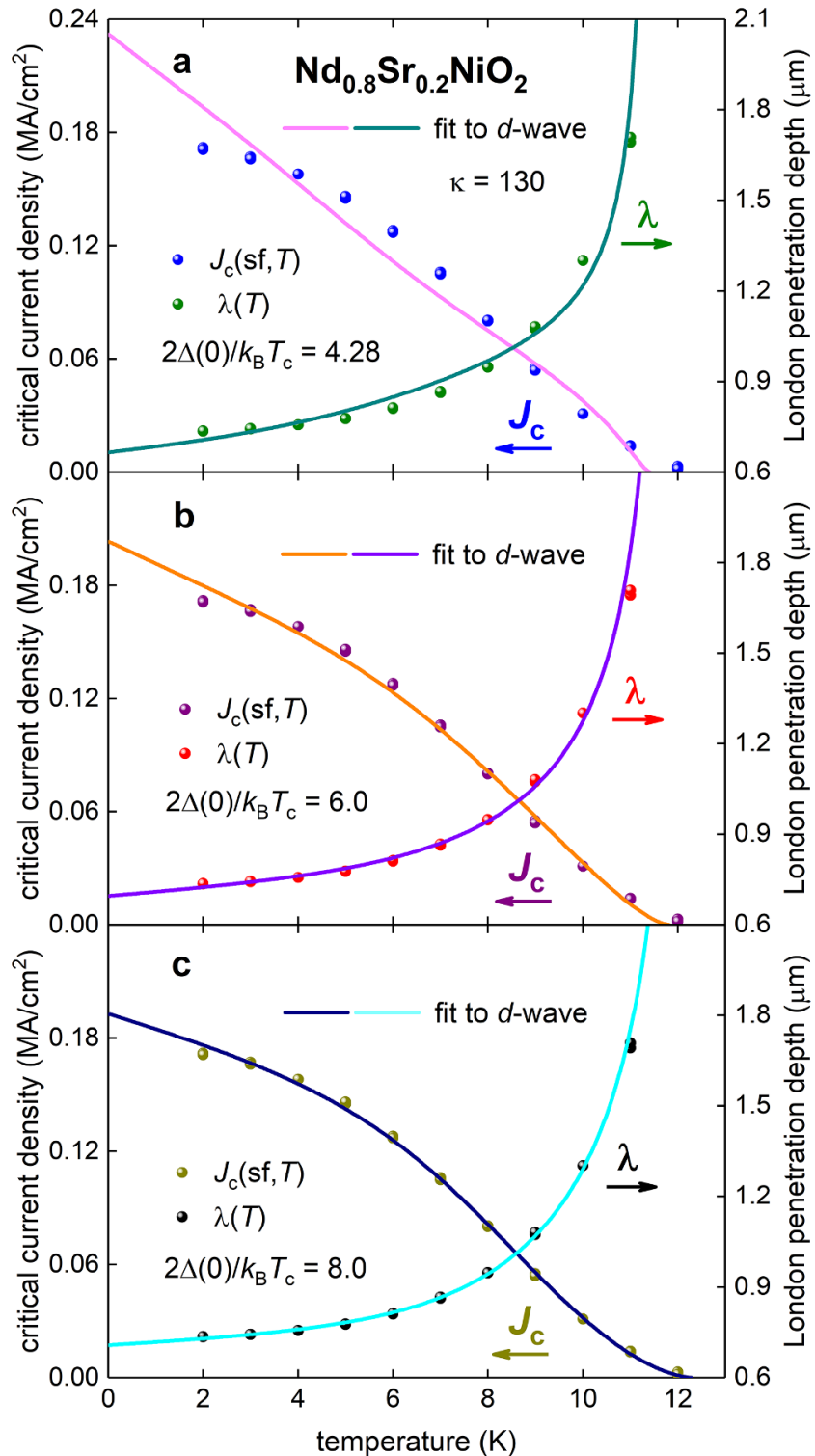

Fig. 4. The self-field critical current density, $J_{\mathrm{c}}(\mathrm{sf}, T)$, for $\mathrm{Nd}_{0.8} \mathrm{Sr}_{0.2} \mathrm{NiO}_{2}$ thin film with raw data processed from the work of Li et al. [3] and data fit to singleband $d$-wave model with three fixed $\frac{2 \Delta(0)}{k_{B} T_{\mathcal{C}}}$ ratios. Ginzburg-Landau parameter $\kappa=130$ is used for all three fits. (a) $\frac{2 \Delta(0)}{k_{B} T_{C}}=4.28$ (fixed), $\Delta C / C=3.2 \pm 1.8$; $\lambda(0)=665 \pm 5 \mathrm{~nm}, T_{\mathrm{c}}=11.4 \mathrm{~K}$, the goodness of fit $R=0.854$; (b) $\frac{2 \Delta(0)}{k_{B} T_{c}}=6.0$ (fixed), $\Delta C / C=1.5 \pm 0.2 ; \lambda(0)=695 \pm 2 \mathrm{~nm}, T_{\mathrm{c}}=11.8 \mathrm{~K}$, the goodness of fit $R=0.981 ;$ (c) $\frac{2 \Delta(0)}{k_{B} T_{C}}=8.0$ (fixed), $\Delta C / C=0.97 \pm 0.07 ; \lambda$ (0) $=708 \pm 1 \mathrm{~nm}, T_{\mathrm{c}}=12.3 \mathrm{~K}$, the goodness of fit $R=0.994$.

of $d$-wave superconductivity; and (c) to $\frac{2 \Delta(0)}{k_{B} T_{c}}=8.0$ which was chosen to be overwhelmingly large. It can be seen in Fig. 4 that for all fits are not consistent with experimental $J_{\mathrm{c}}(\mathrm{sf}, T)$ data. Obviously that fit to $d$-wave pairing symmetry showed in Fig. 3(a) is better matched experimental data, but deduced free-fitting ratio of $\frac{2 \Delta(0)}{k_{B} T_{c}} \approx 1800$ cannot be considered to have physical meaning as we mentioned already above.

By taking in account a good agreement between $\frac{2 \Delta(0)}{k_{B} T_{C}}$ and $\frac{\Delta C}{C}$ values deduced from experimental $B_{\mathrm{c} 2}(T)$ and $J_{\mathrm{c}}(\mathrm{sf}, T)$ data for $\mathrm{Nd}_{0.8} \mathrm{Sr}_{0.2} \mathrm{NiO}_{2}$ in the assumption of $s$-wave symmetry (Eqs. (18) and (19) and Table 1, respectively), which all are within BCS weak-coupling limits for this symmetry, and a fact that $s$-wave pairing symmetry is the most conventional symmetry we can conclude that $\mathrm{Nd}_{0.8} \mathrm{Sr}_{0.2} \mathrm{NiO}_{2}$ nickelate is weak-coupling single band high- $\mathrm{s}$-wave superconductor. 
Table 1

Deduced $2 \Delta(0) / k_{\mathrm{B}} T_{\mathrm{c}}$ and $\Delta C / C$ values for $\mathrm{Nd}_{0.8} \mathrm{Sr}_{0.2} \mathrm{NiO}_{2}$ from $J_{\mathrm{c}}(\mathrm{sf}, T)$ fits to Eqs. (6)-(8) and BCS weak-coupling limits for the same parameters within for $s$-, $d$-, and $p$-wave pairing symmetries [20,23]. For $d$-wave symmetry, $\Delta_{m}(0)$ was used (which is the maximum amplitude of the $k$-dependent $d$-wave gap, $\left.\Delta(\theta)=\Delta_{m}(0) \cos (2 \theta)\right)$.

\begin{tabular}{|c|c|c|c|c|}
\hline $\begin{array}{l}\text { Pairing symmetry } \\
\text { and experiment } \\
\text { geometry }\end{array}$ & Deduced $\frac{2 \Delta(0)}{k_{B} T_{\mathcal{C}}}$ & $\begin{array}{l}\text { BCS weak- } \\
\text { coupling } \\
\text { limit of } \frac{2 \Delta(0)}{k_{B} T_{C}}\end{array}$ & Deduced $\frac{\Delta C}{C}$ & $\begin{array}{l}\text { BCS weak- } \\
\text { coupling } \\
\text { limit of } \frac{\Delta C}{C}\end{array}$ \\
\hline$s$-wave & $3.5 \pm 0.2$ & 3.53 & $1.5 \pm 0.2$ & 1.43 \\
\hline$d$-wave & $1.8 \cdot 10^{3}$ & 4.28 & $0.68 \pm 0.08$ & 0.995 \\
\hline$p$-wave; axial $\mathbf{A} \perp l$ & $4.1 \pm 0.1$ & 4.06 & $1.07 \pm 0.08$ & 1.19 \\
\hline$p$-wave; axial $\mathbf{A} \| \boldsymbol{l}$ & $9.0 \pm 2.4$ & 4.06 & $1.55 \pm 0.05$ & 1.19 \\
\hline $\begin{array}{l}p \text {-wave; polar } \\
\quad \mathbf{A} \perp l\end{array}$ & $1.95 \cdot 10^{3}$ & 4.92 & $2.5 \pm 0.4$ & 0.79 \\
\hline$p$-wave; polar A\|l & $5.3 \pm 0.3$ & 4.92 & $0.63 \pm 0.03$ & 0.79 \\
\hline
\end{tabular}

\section{Conclusions}

Recently discovered [3] an infinite-layer nickelate $\mathrm{Nd}_{0.8} \mathrm{Sr}_{0.2} \mathrm{NiO}_{2}$ superconductor is a new member of bulk oxide superconductors for which experimental $B_{\mathrm{c} 2}(T)$ and $J_{\mathrm{c}}(\mathrm{sf}, T)$ data are analysed in this paper.

In result, it is found that an infinite-layer nickelate $\mathrm{Nd}_{0.8} \mathrm{Sr}_{0.2} \mathrm{NiO}_{2}$ is weak-coupling single band high- $\kappa s$-wave superconductor.

\section{Declaration of Competing Interest}

The author declares that he has no known competing financial interests or personal relationships that could have appeared to influence the work reported in this paper.

\section{Acknowledgement}

Author thanks Dr. W. P. Crump (Aalto University) for invaluable help. Author also thanks financial support provided by the state assignment of Minobrnauki of Russia (theme "Pressure" No. AAAA-A18118020190104-3) and by Act 211 Government of the Russian Federation, contract No. 02.A03.21.0006.

\section{References}

[1] Siegrist T, Zahurak SM, Murphy DW, Roth RS. The parent structure of the layered high-temperature superconductors. Nature 1988;334:231-2.

[2] Azuma M, Hiroi Z, Takano M, Bando Y, Takeda Y. Superconductivity at $110 \mathrm{~K}$ in the infinite-layer compound $\left(\mathrm{Sr}_{1-x} \mathrm{Ca}_{\mathrm{x}}\right)_{1-\mathrm{y}} \mathrm{CuO}_{2}$. Nature 1992;356:775-6.

[3] Li D, Lee K, Wang BY, Osada M, Crossley S, Lee HR, et al. Superconductivity in an infinite-layer nickelate. Nature 2019;572:624-7.

[4] Remeika JP, Geballe TH, Matthias BT, Cooper AS, Hull GW, Kelly EM. Superconductivity in hexagonal tungsten bronzes. Phys Lett A 1967:24:565-6.

[5] Johnston DC, Prakash H, Zachariasen WH, Viswanathan R. High temperature superconductivity in the LiTiO ternary system. Mater Res Bull 1973;8:777-84.

[6] Sleight AW, Gillson JL, Bierstedt PE. High-temperature superconductivity in the $\mathrm{BaPb}_{1-\mathrm{x}} \mathrm{Bi}_{\mathrm{x}} \mathrm{O}_{3}$ systems. Solid State Commun 1975;17:27-8.

[7] Bednorz JG, Müller KA. Possible high Tc superconductivity in the $\mathrm{Ba}-\mathrm{La}-\mathrm{Cu}-\mathrm{O}$ system. Zeitschrift für Physik B Condensed Matter 1986;64:189-93.

[8] Maeno Y, Hashimoto H, Yoshida K, Nishizaki S, Fujita T, Bednorz JG, et al. Superconductivity in a layered perovskite without copper. Nature 1994;372:532-4.

[9] Hirsch JE, Marsiglio F. Hole superconductivity in infinite-layer nickelates. Physica C 2019;566:1353534.

[10] Botana AS, Norman MR. Similarities and differences between infinite-layer nickelates and cuprates and implications for superconductivity; 2019. arXiv:1908. 10946v2.

[11] Jiang M, Berciu M, Sawatzky GA. Doped holes in NdNiO2 and high-Tc cuprates show little similarity; 2019. arXiv:1909.02557.

[12] Nomura Y, et al. Formation of a two-dimensional single-component correlated electron system and band engineering in the nickelate superconductor $\mathrm{NdNiO}_{2}$. Phys. Rev. B 2019;100:205138.

[13] Li Q, He C, Si J, Zhu X, Zhang Y, Wen H-H. Absence of superconductivity in bulk Nd1 - xSrxNiO2; 2019. arXiv:1911.02420v3.

[14] Ginzburg VL, Landau LD. On the theory of superconductivity Zh. Eksp. Teor. Fiz.
1950;20:1064-82

[15] Poole PP, Farach HA, Creswick RJ, Prozorov R. Superconductivity, 2nd ed., London, UK; 2007.

[16] Gor'kov LP. The critical supercooling field in superconductivity theory. Soviet Phys. JETP 1960;10:593-9.

[17] Jones CK, Hulm JK, Chandrasekhar BS. Upper critical field of solid solution alloys of the transition elements. Rev. Mod. Phys. 1964;36:74-6.

[18] Helfand E, Werthamer NR. Temperature and purity dependence of the superconducting critical field, $H_{\mathrm{c} 2}$ II. Phys. Rev. 1966;147:288-94.

[19] Werthamer NR, Helfand E, Hohenberg PC. Temperature and purity dependence of the superconducting critical field, $H_{\mathrm{c} 2}$. III. Electron spin and spin-orbit effects. Phys Rev 1966;147:295-302.

[20] Baumgartner T, Eisterer M, Weber HW, Fluekiger R, Scheuerlein C, Bottura L. Effects of neutron irradiation on pinning force scaling in state-of-the- $\mathrm{art}_{\mathrm{Nb}} \mathrm{Sn}$ wires. Supercond Sci Technol 2014;27:015005.

[21] Talantsev EF. Classifying superconductivity in compressed $\mathrm{H}_{3} \mathrm{~S}$. Mod Phys Lett B 2019;33:1950195.

[22] Gross F, Chandrasekhar BS, Einzel D, Andres K, Hirschfeld PJ, Ott HR, et al Anomalous temperature dependence of the magnetic field penetration depth in superconducting $\mathrm{UBe}_{3}$. Z Phys B - Condensed Matter 1986;64:175-88.

[23] Talantsev EF. Critical de Broglie wavelength in superconductors. Mod Phys Lett B 2018;32:1850114.

[24] Talantsev EF, Crump WP, Island JO, Xing Y, Sun Y, Wang J, Tallon JL. On the origin of critical temperature enhancement in atomically thin superconductors. 2D Mater 2017;4:025072

[25] Gross-Alltag F, Chandrasekhar BS, Einzel D, Hirschfeld PJ, Andres K. London field penetration in heavy fermion superconductors Z. Phys. B - Condensed Matter 1991;82:243-55

[26] Talantsev EF, Iida K, Ohmura T, Matsumoto T, Crump WP, Strickland NM, et al. P. wave superconductivity in iron-based superconductors. Sci Rep 2019;9:14245.

[27] Talantsev EF, Mataira RC, Crump WP. Classifying superconductivity in in Moiré graphene superlattices. Sci Rep 2020;10:212.

[28] Fête A, Rossi L, Augieri A, Senatore C. Ionic liquid gating of ultra-thin $\mathrm{YBa}_{2} \mathrm{Cu}_{3} \mathrm{O}_{7}$ films. Appl Phys Lett 2016;109:192601.

[29] Liu C, et al. Two-dimensional superconductivity and topological states in $\mathrm{PdTe}_{2}$ thin films. Phys Rev Mater 2018;2:094001.

[30] Qu D-X, Teslich NE, Dai Z, Chapline GF, Schenkel T, Durham SR, et al. Onset of a two-dimensional superconducting phase in a topological-insulator-normal-metal $\mathrm{Bi}_{1-\mathrm{x}} \mathrm{Sb}_{\mathrm{x}} / \mathrm{Pt}$ junction fabricated by ion-beam techniques. Phys Rev Lett 2018;121:037001.

[31] Pal B, et al. Experimental evidence of a very thin superconducting layer in epitaxial indium nitride. Supercond Sci Technol 2019;32:015009.

[32] Zheliuk O, Lu JM, Chen QH, El Yumin AA, Golightly S, Ye JT. Josephson coupled Ising pairing induced in suspended $\mathrm{MoS}_{2}$ bilayers by double-side ionic gating. Nat Nanotechnol 2019;14:1123-8.

[33] Jovanović VP, Li ZZ, Raffy H, Briatico J, Sinchenko AA, Monceau P. Resistive upper critical fields and anisotropy of an electron-doped infinite-layer cuprate. Phys Rev B 2009;80:024501.

[34] Bardeen J, Cooper LN, Schrieffer JR. Theory of superconductivity. Phys Rev 1957;108:1175-204.

[35] Wen CHP, et al. Unveiling the superconducting mechanism of $\mathrm{Ba}_{0.51} \mathrm{~K}_{0.49} \mathrm{BiO}_{3}$. Phys Rev Lett 2018;121:117002.

[36] Talantsev EF. Classifying induced superconductivity in atomically thin Dirac-cone materials. Condensed Matter 2019;4:83.

[37] Fruchter L, Jovanovic V, Raffy H, Sid Labdi, Bouquet F, Li ZZ. Penetration depth of electron-doped infinite-layer $\mathrm{Sr}_{0.88} \mathrm{La}_{0.12} \mathrm{CuO}_{2+\mathrm{x}}$ thin films. Phys Rev B 2010;82:144529.

[38] Puźniak R, Usami R, Isawa K, Yamauchi H. Superconducting-state thermodynamic parameters and anisotropy of $\mathrm{HgBa}_{2} \mathrm{Ca}_{n-1} \mathrm{Cu}_{n} \mathrm{O}_{\mathrm{y}}$ by reversible magnetization measurements. Phys Rev B 1995;52:3756-64.

[39] Hosono H, et al. Exploration of new superconductors and functional materials, and fabrication of superconducting tapes and wires of iron pnictides. Sci Technol Adv Mater 2015;16:033503.

[40] Iida Kazumasa, Hänisch Jens, Tarantini Chiara. Fe-based superconducting thin films on metallic substrates: growth, characteristics, and relevant properties. Appl Phys Rev 2018;5(3):031304. https://doi.org/10.1063/1.5032258.

[41] Kauffmann-Weiss S, et al. Microscopic origin of highly enhanced current carrying capabilities of thin NdFeAs(O, F) films. Nanoscale Adv 2019;1:147. https://doi.org/ 10.1039/C9NA00147F.

[42] Hänisch J, et al. Fe-based superconducting thin films - preparation and tuning of superconducting properties. Supercond Sci Technol 2019;32:093001.

[43] Hosono H, Kuroki K. Iron-based superconductors: current status of materials and pairing mechanism. Physica C 2015;514:399-422.

[44] Khasanov R, et al. Nodeless superconductivity in the infinite-layer electron-doped cuprate superconductor $\mathrm{Sr}_{0.9} \mathrm{La}_{0.1} \mathrm{CuO}_{2}$. Phys Rev B 2008;77:184512.

[45] Uemura YJ, et al. Basic similarities among cuprate, bismuthate, organic, Chevrelphase, and heavy-fermion superconductors shown by penetration-depth measurements. Phys Rev Lett 1991;66:2665-8.

[46] Uemura YJ. Dynamic superconductivity responses in photoexcited optical conductivity and Nernst effect. Phys Rev Mater 2019;3:104801.

[47] Won H, Maki K. $d$-wave superconductor as a model of high- $T_{\mathrm{c}}$ superconductors. Phys Rev B 1994;49:1397-402. 